\title{
Computational Fluid-Structure Interaction of a Deformable Flapping Wing for Micro Air Vehicle Applications
}

\author{
Jian Tang ${ }^{* 1}$, Satish Chimakurthi ${ }^{2}$, Rafael Palacios ${ }^{*}{ }^{3}$, Carlos E.S. Cesnik $*^{4}$, and Wei Shyy $*^{5}$ \\ * Department of Aerospace Engineering \\ University of Michigan, Ann Arbor, MI, 48109, U.S.A. \\ ** Department of Aeronautics \\ Imperial College, London, SW7 2AZ, UK
}

\begin{abstract}
Motivated by micro air vehicle applications, a fluid-structure coupling procedure between a NavierStokes solver and a three-dimensional FEM beam solver is presented along with selected results highlighting some of the aerodynamics implications. The fluid model includes laminar, the $k-\varepsilon$ turbulence closure, and a filter-based $k-\varepsilon$ closure. The structural model is based on an asymptotic approximation to the equations of elasticity. Using the slenderness as the small parameter, the equations are decomposed into two independent variational problems, corresponding to (i) crosssectional, small-deformation and (ii) longitudinal, large deformation analyses. A model example problem corresponding to a NACA0012 wing of aspect ratio 3 in pure heave motion is presented and the results compared against available experiment data. Quantitative comparisons with experiment are done for the rigid wing and the implications of wing flexibility on aerodynamics are presented in a qualitative sense. It was observed that phase lag of the wing tip displacement relative to the flapping motion becomes more pronounced as the fluid density increases.
\end{abstract}

\section{Nomenclature}

$\begin{array}{ll}a_{\text {root }} & \text { Plunge amplitude } \\ \mathrm{A}_{\mathrm{cs}} & \text { Area of a typical cross-section of the beam structure } \\ b & \text { Semi chord } \\ c & \text { Chord length } \\ C_{T} & \text { Horizontal force coefficient } \\ C_{y} & \text { Vertical force coefficient } \\ \mathrm{E} & \text { Young's modulus } \\ \mathrm{f}_{\mathrm{B}} & \text { 3x1 matrix of external (aerodynamic) forces at a point on the beam reference line expressed } \\ F_{x} & \text { in B frame } \\ F_{y} & \text { Horizontal force acting on the airfoil } \\ \mathrm{h}_{\mathrm{r}} & \text { Vertical force acting on the airfoil } \\ & \text { Non-dimensional plunge amplitude }=\frac{a_{r o o t}}{C}\end{array}$

\footnotetext{
${ }^{1}$ Post doctoral research fellow, jiantang@umich.edu, Member AIAA

${ }^{2}$ Doctoral candidate, satishkc@umich.edu, Member AIAA

${ }^{3}$ Lecturer, r.palacios-nieto@imperial.ac.uk, Member AIAA

${ }^{4}$ Associate professor, cesnik@umich.edu, Associate Fellow AIAA

${ }^{5}$ Clarence L. "Kelly” Johnson Collegiate Professor and Chair, weishyy@umich.edu, Fellow AIAA
} 


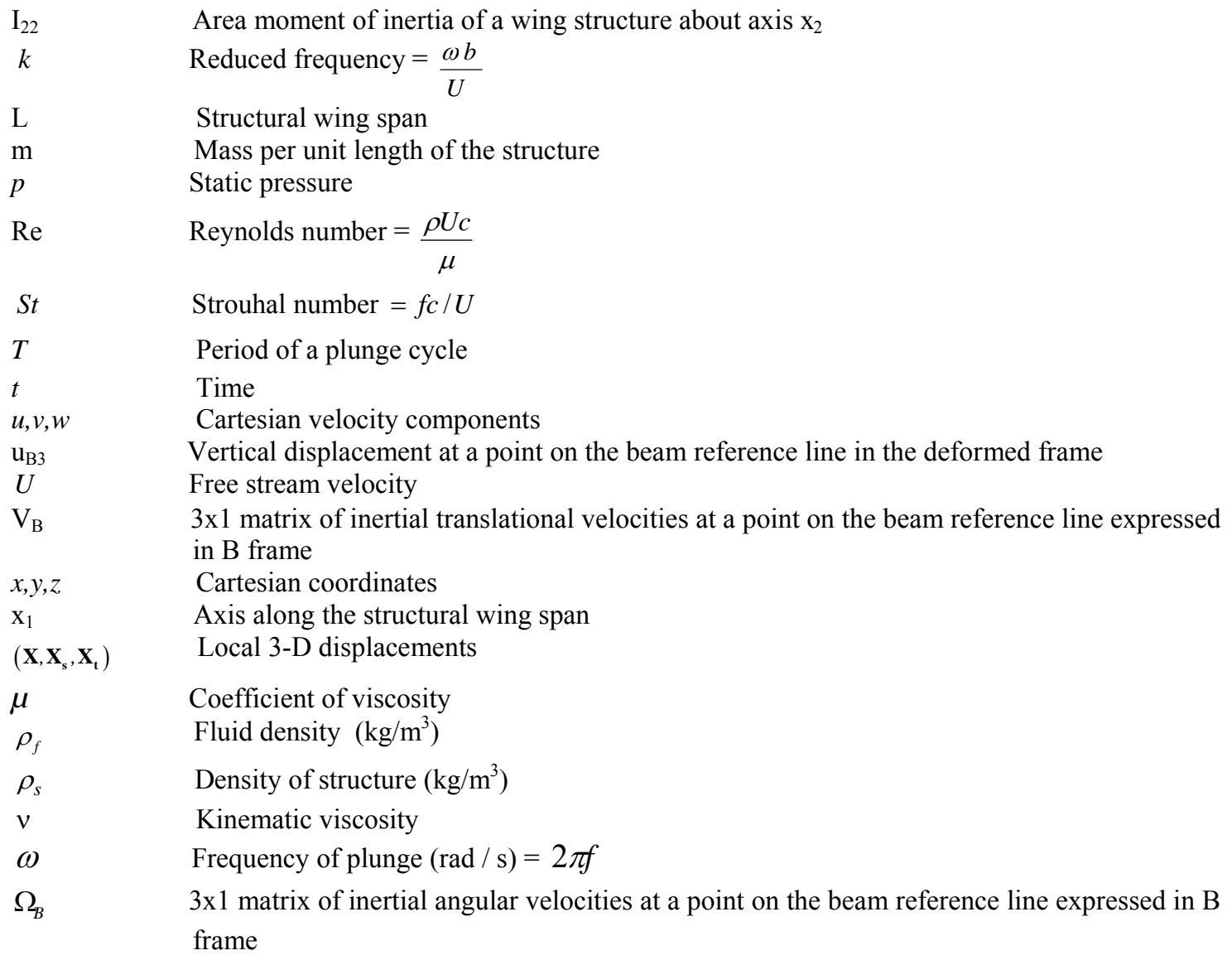

Superscript

non-dimensional quantities

\section{Introduction}

$\mathrm{I}_{\mathrm{o}}^{\mathrm{n}}$ recent years, with rising interests in micro air vehicles and in smaller nano air vehicles, numerous experimental and computational studies in the area of aerodynamic force generation by flapping flight have been reported, e.g., [1-7]. Most of the previous work focused on the lift generation mechanisms: clapand-fling mechanism ${ }^{1}$, delayed stall phenomenon associated with leading-edge vortices ${ }^{2}$, fast pitch-up ${ }^{3}$, and wake-capturing ${ }^{3}$. Van den Berg and Ellington ${ }^{4}$ and Ellington et $\mathrm{al}^{2}$ found the leading-edge vortex and the spanwise flow above a hawkmoth's wing. Liu and Kawachi ${ }^{5}$ as well as Shyy and Liu ${ }^{6}$ conducted unsteady Navier-Stokes simulations to highlight the aerodynamics and fluid physics associated with the leading-edge vortex and the spanwise flow. Dickinson et al. ${ }^{3}$ identified the force peaks generated during fast pitch-up of a flapping wing at the end of a stroke and the wake-capture mechanism in the experiments. These phenomena were studied in the numerical simulations conducted by Sun and Tang ${ }^{7}$ and Ramamurti and Sandberg ${ }^{8}$. Sun and Tang ${ }^{7}$ provided a different perspective for the understanding of these phenomena from that of Dickinson et $\mathrm{al}^{3}$. Viieru et al. ${ }^{9}$ and Tang et al. ${ }^{10}$ investigated the lift generation mechanism by simulating two flapping modes (normal hovering ${ }^{11}$ and water treading mode ${ }^{12}$ ) of an airfoil section. 
Different force patterns between the two hovering modes were reported in their work. A wake-capturing mechanism is identified by small lift peaks at the beginning of the stroke after the stroke reversal in the normal hovering mode. Also, for the normal hovering mode as the Reynolds number increases, a distinct asymmetrical force pattern between forward and backward strokes is noticed at small flapping amplitudes. In the case of the water treading mode, the lift force variation shows a symmetric pattern between forward and backward strokes. Shyy et $\mathrm{al}^{13}$ have offered a detailed review of the current state of the knowledge in all of these aspects.

With the synergy generated between the long standing interest on this subject in the biological research community and the efforts being made in the aerospace engineering and science community, substantial progress has taken place on numerous fronts related to flapping wing aerodynamics, propulsion, and fluidstructure interactions, and control issues. In this study, we report our ongoing efforts in developing fluidstructural interaction simulation capabilities. Insect, bat and bird wings have varied morphology; furthermore, their composite structural components result in substantial degrees of anisotropy in the wing's overall mechanical properties. Combes and Daniel ${ }^{14}$ conducted a study on the structure of the insect wings. Based on their measurements, the spanwise stiffness of the insect wings they studied is about 1-2 orders larger than that of the chord-wise. Wootton et $\mathrm{al}^{15}$ numerically modeled insect-wings by the finite element method. Heathcote et al. ${ }^{16,1718}$ experimentally investigated the effects of stiffness on thrust generation of an airfoil undergoing a plunging motion under various free stream velocities. Direct force measurements showed that the thrust/input-power ratio was found to be greater for the flexible airfoils than a rigid airfoil. They also observed that at high plunging frequencies, the medium flexible airfoil generates the largest thrust, while the most flexible airfoil generates the most thrust at low frequencies. To study the effect of the spanwise stiffness on the thrust, lift and propulsive efficiency of a plunging wing, a water tunnel study was conducted by them on a NACA0012 uniform wing of aspect ratio 3. They observed that for Strouhal numbers greater than 0.2 , a degree of spanwise flexibility was found to be beneficial..

In a previous effort, Tang et $\mathrm{al}^{19}$ conducted a two dimensional fluid-structure coupled simulation to study a flexible airfoil plunging in forward flight. A 2D finite element formulation using beam elements was utilized to couple with an incompressible flow solver via an implicit method. They proposed that passive pitching which is the deformation caused by the fluid pressure on the airfoil changes the effective angle of attack, causing noticeable differences to lift and thrust generated. Furthermore, as evidenced in the case study of the prescribed shape change to the airfoil, even at $\mathrm{Re}=100$, in the plunging motion, the force acting on airfoil was dominated by pressure and the viscous force was of little impact to the overall aerodynamic outcome. Detailed airfoil shape is secondary compared to the equivalent angle of attack.

In this paper, a high-fidelity computational framework for fluid-structure interactions of flapping wing MAVs is presented. Preliminary results are obtained on 3D rigid and flexible wings in pure plunge motion and compared against the experimental results of Heathcote et $\mathrm{al}^{18}$. The main objectives of this paper are: (i) to explain the development of a computational framework for fluid-structure interactions of flapping wing MAVs (ii) to assess the aerodynamics implications of rigid and flexible wings based on the experimental results of Heathcote et $\mathrm{al}^{18}$ and (iii) to probe into the effect of turbulence closures on the fluid dynamics of a rigid wing in plunge. 


\section{Numerical methodology}

\section{A. Flow solver (STREAM)}

The fluid solution is obtained from the incompressible Navier-Stokes equations and the continuity equation

$$
\begin{aligned}
\frac{\partial u_{i}}{\partial t}+\frac{\partial}{\partial x_{j}}\left(u_{j} u_{i}\right) & =-\frac{1}{\rho_{f}} \frac{\partial p}{\partial x_{i}}+\left(v+v_{t}\right) \frac{\partial^{2} u_{i}}{\partial x_{j}^{2}} \\
\frac{\partial u_{j}}{\partial x_{j}} & =0
\end{aligned}
$$

where $\rho_{f}$ is the fluid density, $u_{i}$ is the velocity vector, $t$ is the time, $x_{i}$ is the position vector, $p$ is the pressure, $v$ is the kinematic viscosity and $v_{t}$ is the turbulence viscosity.

Based on the definition of the motion ${ }^{19}$ for forward flight, if the free stream velocity $(U)$, the chord length (c) and the inverse plunging/pitching (1/f) frequency are used as the velocity, length and time scales respectively, the Reynolds and Strouhal numbers appear as $\mathrm{Re}=U_{c} / v, \mathrm{Re}_{t}=U_{C} / v_{t}$ and $S t=f_{C} / U$. With these choices of the scaling parameters, the non-dimensional form of the Navier-Stokes equations become:

$$
\mathrm{St} \frac{\partial}{\partial \bar{t}}\left(\bar{u}_{i}\right)+\frac{\partial}{\partial \bar{x}_{j}}\left(\bar{u}_{j} \bar{u}_{i}\right)=-\frac{\partial \bar{p}}{\partial \bar{x}_{i}}+\left(\frac{1}{\operatorname{Re}}+\frac{1}{\operatorname{Re}_{t}}\right) \frac{\partial^{2} \bar{u}_{i}}{\partial \bar{x}_{j}^{2}}
$$

It should be noted that the relation between Strouhal number and reduced frequency is $k=\frac{\omega b}{U}=h_{r} S t$. This relation is used to get the plunging frequency from the experiment data. At the Reynolds number of $3 \times 10^{4}$ which is of interest in this work, depending on the flapping motions, wing flexibility, and free stream conditions, the flow field is likely to exhibit laminar, transition, and turbulent characteristics. We adopted three approaches in our fluid models: (i) strictly laminar flow computations, with no additional models other than the Navier-Stokes equations, (ii) the original k- $\varepsilon$ two-equation model ${ }^{20}$, and (iii) a filter-based k- $\varepsilon$ model, reported by Johanson et $\mathrm{al}^{21}$. The $\mathrm{k}-\varepsilon$ two-equation model was originally developed for high Reynolds number, stationary flows. The filter model allows the computations to use reduced amount of eddy viscosity whenever there is an adequate numerical resolution, so that the flow field doesn't experience excessive damping caused by eddy viscosity. As demonstrated by Johanson et $\mathrm{al}^{21}$, for flows at modest Reynolds numbers, the filter model can offer improved predictive capability than those of the RANSbased engineering turbulence closures.

The equations for the k- $\varepsilon$ two-equation model employed in the computations in this work are presented below in Eqn 3.

$$
\begin{gathered}
\frac{\partial k}{\partial t}+\frac{\partial\left(u_{j} k\right)}{\partial x_{j}}=\frac{P_{t}}{\rho_{f}}-\varepsilon+\frac{\partial}{\partial x_{j}}\left[\left(v+\frac{v_{t}}{\sigma_{k}}\right) \frac{\partial k}{\partial x_{j}}\right] \\
\frac{\partial \varepsilon}{\partial t}+\frac{\partial\left(u_{j} \varepsilon\right)}{\partial x_{j}}=C_{\varepsilon 1} \frac{\varepsilon}{k} \frac{P_{t}}{\rho_{f}}-C_{\varepsilon 2} \frac{\varepsilon^{2}}{k}+\frac{\partial}{\partial x_{j}}\left[\left(v+\frac{v_{t}}{\sigma_{\varepsilon}}\right) \frac{\partial \varepsilon}{\partial x_{j}}\right]
\end{gathered}
$$

The filter model reported by Johanson et $\mathrm{al}^{21}$ is realized by imposing a filter on the $\mathrm{k}-\varepsilon$ turbulence model. By integrating the Kolmogorov model for the speed and energy spectrum in the sub-filter flow, a viscosity model is obtained and is shown in Eqn 4 below. 


$$
v_{t}=C_{\mu} \cdot \operatorname{Min}\left[1, C_{3} \frac{\Delta \cdot \varepsilon}{k^{3 / 2}}\right] \cdot \frac{k^{2}}{\varepsilon}
$$

Where, $C_{\mu}$ is $0.09, C_{3}$ is 1.0 and $\Delta$ is the filter size.

The numerical solution is based on the extension of the original SIMPLE family of algorithms ${ }^{22}$, with an employment of the cartesian and contravariant velocity variables to facilitate strong conservation law formulations and consistent finite volume treatment ${ }^{23}$. The convection terms are discretized using a secondorder upwind scheme, while the pressure and viscous terms with a second-order central difference scheme. For time integration, an implicit second order upwind scheme is employed. A moving grid technique employing the master-slave concept ${ }^{24}$ is used to re-mesh the multi-block structured grid for fluid-structure interaction problems. The geometric conservation law (GCL) originally proposed by Thomas and Lombard $^{25}$ was incorporated to consistently compute the cell volume in a moving boundary problem and eliminate the artificial mass sources. The specific implementation and implications of the GCL in the context of the present solution algorithm have been discussed by Shyy et $\mathrm{al}^{26}$.

\section{B. Structural solver (UM/NLABS)}

The geometrically-nonlinear structural dynamic solution is based on an asymptotic approach to the equations governing the dynamics of a general 3-D anisotropic slender solid ${ }^{27,28}$. The presence of a small parameter (the inverse of the wing aspect ratio) defines a multi-scale solution process, in which the problem is decomposed into independent cross-sectional (small-scale) and longitudinal (long-scale) analyses. The longitudinal problem solves for average measures of deformation of the reference line under given external excitations. The cross-sectional problem solves the local deformation for given values of the long-scale variables. Both problems are tightly coupled and together provide an approximation to the displacement field in the original 3-D domain, as shown in Fig 1a.

To derive the three-dimensional dynamic equations of a slender solid, consider a slender solid of slowly-varying cross section depicted in Fig 1b. The characteristic lengths involved in its deformation are: 1) the cross-sectional characteristic dimension, $h$; 2) the characteristic wave length of the response along the longitudinal dimension, $L$ (depending on the longitudinal rate of change of the geometrical and material properties, on the typical wave length of the excitation, and on the characteristic length of the initial curvature and twist). The assumption of slenderness can be set to $h / L<<1$. One can then define a reference line $r$ along that direction on the undeformed solid, defined by the curvilinear coordinate $x_{1}$, which then transforms into the deformed line $R$. In addition to this, a local coordinate system $b$, given by the base vectors $\left\{\boldsymbol{b}_{\boldsymbol{i}}\left(x_{1}\right)\right\}$, is also defined on each point along the undeformed line, which transforms into a deformed frame $B$, with base vectors $\left\{\boldsymbol{B}_{\boldsymbol{i}}\left(x_{1}\right)\right\}$, (using a Lagrangian representation of the structural deformation). Finally, a global reference frame $a$, with base vectors $\left\{\boldsymbol{a}_{\boldsymbol{i}}\left(x_{1}\right)\right\}$ whose motion is always known with respect to an inertial frame, is also defined. The equations of motion of this slender body are given by Hamilton's principle applied in a time interval $\left[t_{1}, t_{2}\right]$ and in the spatial domain $\Omega$, as

$$
\int_{t_{1}}^{t_{2}}\left\{\int_{\Omega}[\delta(k-u)+\delta w] d \boldsymbol{x}\right\} d t=\delta A
$$




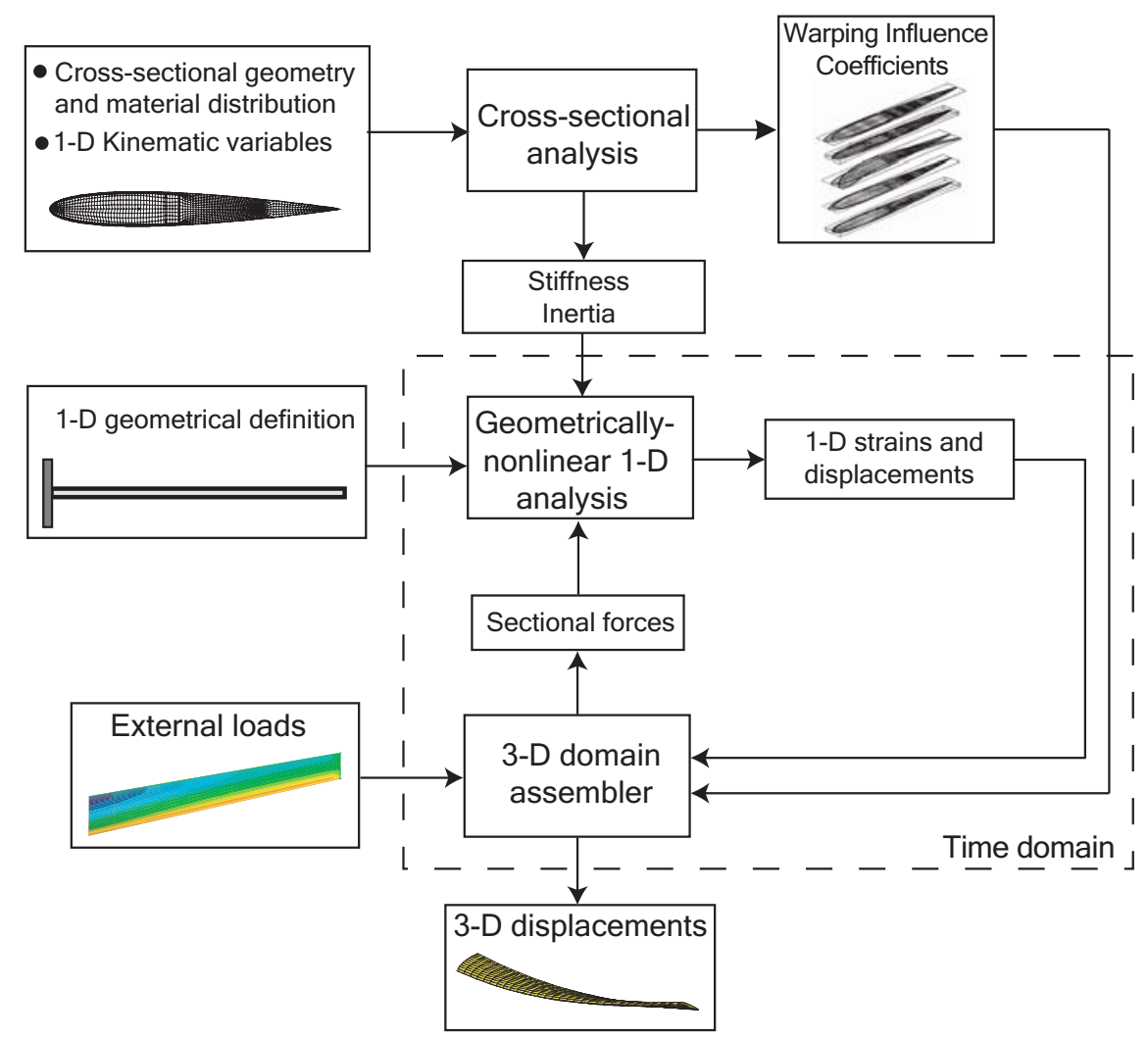

Fig 1a: Asymptotic solution process for 3-D slender structures

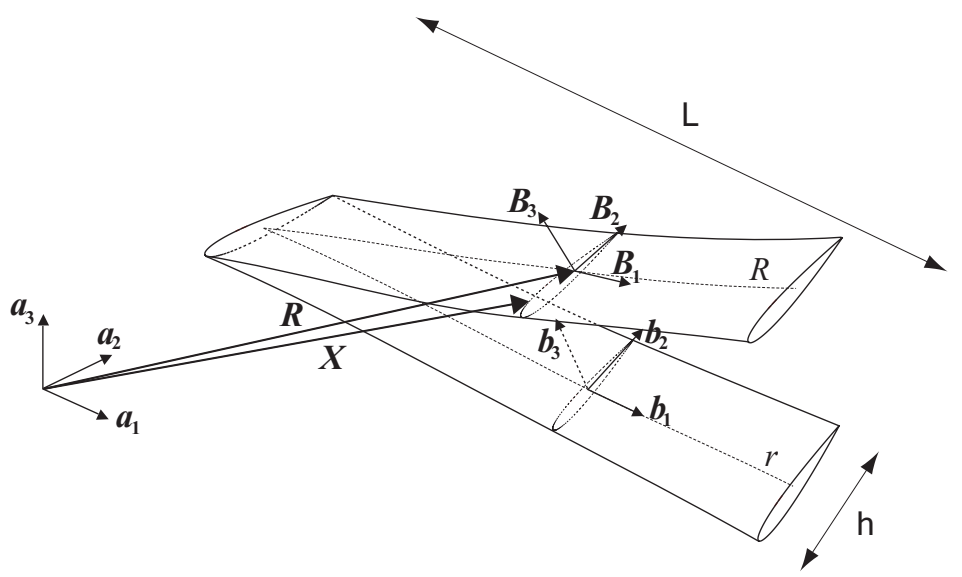

Fig 1b: Coordinate frames in the deformation of a slender solid

where $\delta w$ is the virtual work density of the external forces, $\delta A$ includes any virtual action on the boundaries of the domain $\Omega$ and at the ends of the time interval, and $k$ and $u$ are the kinetic and strain energy densities, respectively. Substitution of the kinetic and strain energy densities in the Hamilton's 
principle and subsequent integration by parts in both time and space variables results in the intrinsic form of the geometrically-nonlinear equations describing the dynamics of a member expressed in the B frame (dimensional) which are included in Eqns 6 and 7.

$m\left(\frac{d}{d t}+\tilde{\Omega}_{B}\right) V_{B}=\left(\frac{d}{d x_{1}}+\tilde{K}_{B}\right) F_{B}+f_{B}$

$\left(\frac{d}{d t}+\tilde{\Omega}_{B}\right)\left(J \Omega_{B}\right)=\left(\frac{d}{d x_{1}}+\tilde{K}_{B}\right) M_{B}+\left(\tilde{e_{1}}+\tilde{\gamma}\right) F_{B}+m_{B}$

In order to identify the fluid-structure scaling parameters, a linear form of the structural governing equations may first be obtained for the special case in which the beam reference line is located at the centroid. Also, the rotary inertia and all the nonlinear contributions are neglected. With these assumptions eqn 7 reduces to eqn 8 .

$$
M_{B}^{\prime}=-\tilde{e_{1}} F_{B}
$$

where $\tilde{e}_{1}=\left[\begin{array}{ccc}0 & 0 & 0 \\ 0 & 0 & -1 \\ 0 & 1 & 0\end{array}\right]$

Eqn 8 is substituted into eqn 6 and the nonlinear terms are neglected. From the reduced form of the set of equations 6 , only the equation concerning the case of bending about axis $B_{2}$ is included in eqn 9 .

$$
m \frac{d V_{B_{3}}}{d t}+\frac{d^{2} M_{B_{2}}}{d x_{1}^{2}}=f_{B_{3}}
$$

This is the familiar equation for bending of an Euler-Bernouli beam about an axis ( $B_{2}$ here) where $m=\rho_{s} A_{c s}$ and $M_{B 2}=E I_{22} \kappa_{2}$. Eqn 9 may be non-dimensionalized by dividing it throughout by a suitable factor which may be appropriately chosen as $\rho_{f} U^{2} c$ in this context. The resulting non-dimensional form of eqn 9 is included in eqn 10. 


$$
\bar{\rho} \overline{\left(A_{c S} L\right)} \frac{d \bar{V}_{B 3}}{d \bar{t}}+\bar{E} I_{22}^{-} \frac{d^{4} \bar{u}_{B 3}}{d \bar{x}_{1}}=\overline{f_{B 3}}
$$

where the non-dimensional parameters are defined as:

$$
\bar{\rho}=\frac{\rho_{s}}{\rho_{f}} \quad \bar{E}=\frac{E}{\rho_{f} U^{2}} \quad \bar{x}_{1}=\frac{x_{1}}{c} \quad \bar{u}_{B 3}=\frac{u_{B 3}}{c} \quad \bar{I}_{22}=\frac{I_{22}}{c^{4}} \quad \bar{t}=\frac{t U}{c} \quad \bar{f}_{B 3}=\frac{f_{B 3}}{\rho_{f} U^{2} C}
$$

Variations of the flow density are considered for the flexible wing computations in this work. That, in effect, varies the density ratio parameter $\bar{\rho}$.

\section{CFD-CSD Coupling}

In this work, a closely-coupled methodology ${ }^{29}$ has been applied to couple the individual CFD and CSD components (Fig. 2). A dedicated interface module was developed to enable communication between the flow and the structure at the wet surface. In the interface module, both the fluid and the structural modules are called alternatively according to the coupling method adopted for the problem. In the case of the explicit coupling method, both the solvers are called once per coupled time-step while exchanging data at the interface. In the case of the implicit coupling method, several sub-iterations are performed between the fluid and the structural solutions within a coupled time-step. Between any two fluid-structure sub-iterations, the initial conditions in the solvers are not updated and hence an updated solution is obtained for the same time-step. This is unlike between the last fluid-structure sub-iteration of a coupled time-step and the first fluid-structure sub-iteration of the subsequent coupled time-step wherein the initial conditions in the solvers are updated and hence the solution is time-marched.

Several interface subroutines have been written to couple UM/NLABS and STREAM including those to control the flow of the coupled solution and to perform interpolation of physical quantities from the CFD to CSD grids and vice-versa via thin-plate spline $e^{29}$ / bilinear interpolation methods. Since both UM/NLABS and STREAM were developed in-house and the source code for both of these solvers is available, a coupled code was achieved simply by compiling the object files of both of the individual solvers along with those of the interface routines to produce a shared executable. UM/NLABS is written in Fortran 90 and STREAM in Fortran 90 / C++. The coupling method chosen for the computations in this work is explicit.

\section{Results and Discussion}

This section is divided into 4 subsections. In the first subsection, a brief description of the test problem that is considered in this work is provided. In the second subsection, details of the fluid and the structural computational models are provided. In the third subsection, computational results on the rigid wing configuration are reported and compared against the experimental results of Heathcote et $\mathrm{al}^{18}$. In the fourth 
sub-section, computational results on the flexible wing configuration are reported at three different flow densities (air density (AD), 10xAD, 416xAD). Experimental results at 813xAD are included along with the lower density computational results to indicate the trends in the thrust coefficient response.

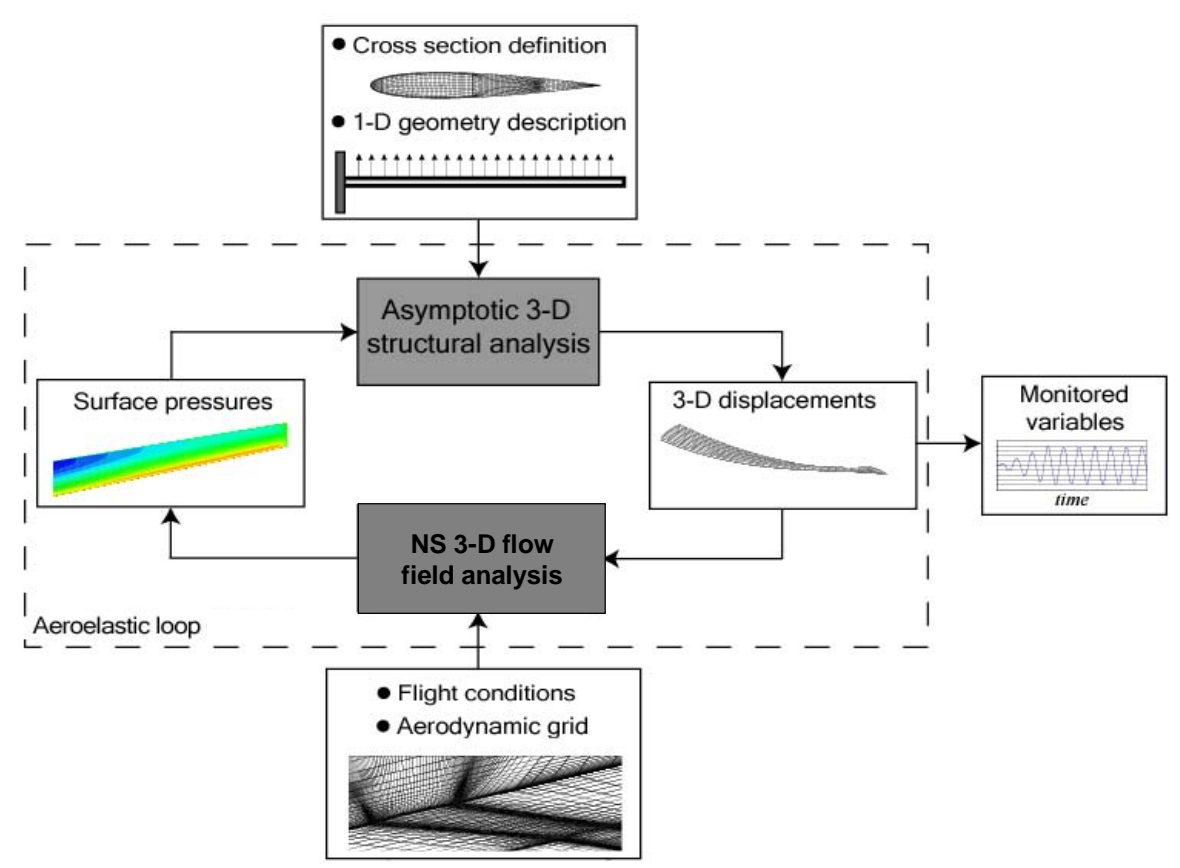

Fig 2: Schematic of the computational framework for flexible wing aerodynamics

\section{A. Problem description}

In an attempt to test the coupled fluid-structure framework discussed previously, preliminary results were obtained on a three-dimensional rectangular wing of NACA0012 uniform cross-section oscillating in pure heave. Water tunnel studies have been performed by Heathcote et $\mathrm{a}^{18}$ to study the effect of span-wise flexibility on the thrust, lift, and propulsive efficiency on this wing configuration. Three wings of $0.3 \mathrm{~m}$ span, $0.1 \mathrm{~m}$ chord with varying levels of flexibility were constructed in that experiment which are shown in

Fig 3. A plunge displacement profile $s=a_{\text {root }} \cos (\omega t)$ is prescribed to the root of the wing at the leading edge.

Overall wing thrust coefficient and the tip displacement response were measured in the experiment amongst other things. Only the 'Rigid', and 'Flexible' versions of the wings highlighted in Fig. 3 were considered in this work.

\section{B. Computational models}

A structural multi-block C-type grid of a NACA0012 wing of aspect ratio 3 was used for the CFD simulations (Fig. 4 - left). Grid size is 120, 56, and 60 in the tangential, radial and spanwise directions 
respectively. Grid sensitivity studies have been performed to identify a grid suitable for the computations in this work.

The structural model for the computations (Fig. 4 - right) is based on a 1-D beam finite-element discretization with 39 elements along the semi-span. Chord-wise deformation is reported as negligible in the experiment, and hence, a beam model with six elastic degrees of freedom, corresponding to extension, twist, and shear and bending in two directions is justified. The beam reference line (cantilevered to a plunging frame of reference) is chosen along the leading edge of the wing and cross-sectional properties are evaluated with respect to the leading edge point. The properties are uniform throughout the semi-span. Since the contribution of the PDMS rubber material to the overall mass and stiffness properties is negligible, for the

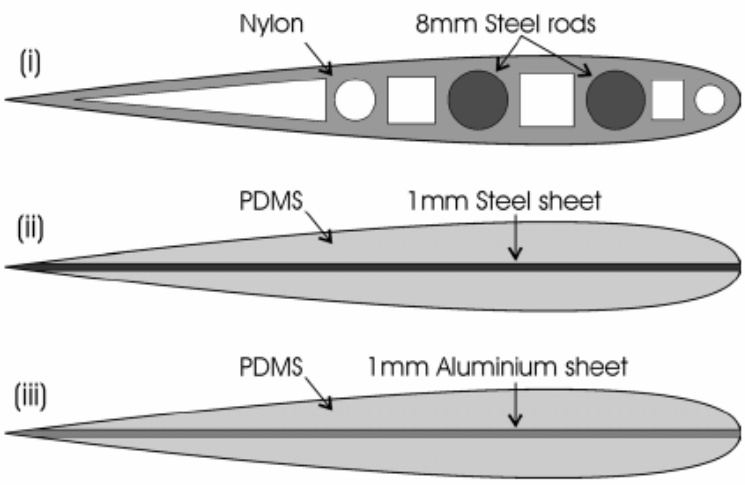

Fig 3: The cross-sections of the 'Rigid (top)', 'Flexible (center)', and 'Very Flexible (bottom) wings used in the experiments of Heathcote et al $^{18}$

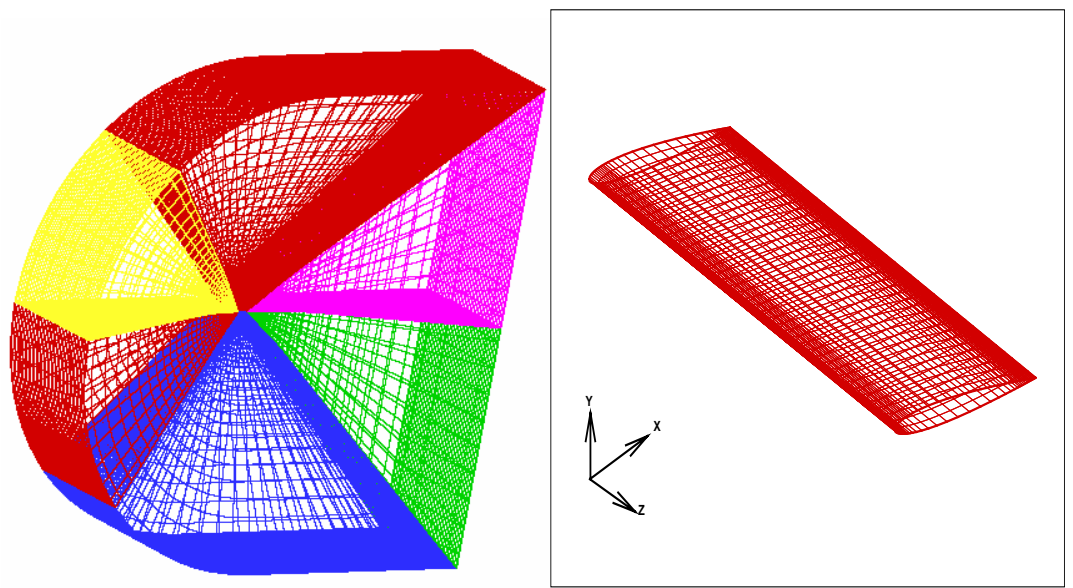

Fig 4: The topology of the grid distribution of the rectangular wing in the CFD computation. (left) The interface grid between the CFD and CSD solver. (right)

evaluation of cross-sectional properties, only the stainless steel stiffener is retained. The 3-D structural solution is obtained by using 75 recovery nodes on each cross section (defined at a 1-D FE node) and hence 
a structured grid of 3000 interface points define the solid side of the aeroelastic interface. For the flexible wing computations in this work, as mentioned previously, only the 'Flexible' wing version of the crosssections used in the experiment was considered.

For the rigid wing computations, the Reynolds number (based on chord) was set to 30,000 and the reduced frequency (based on semi-chord) to 1.82. For the flexible wing computations, variations in flow density were considered while the Reynolds number and the reduced frequency were kept constant. A summary of the mechanical and flow properties are included in Table 1.

\begin{tabular}{|l|c|l|c|}
\hline \multicolumn{4}{|c|}{ Table 1. Wing model parameters and flight conditions } \\
\hline Semispan & $0.3 \mathrm{~m}$ & Reduced frequency - $\mathrm{k}$ & 1.82 \\
\hline Chord (c) & $0.1 \mathrm{~m}$ & Flow velocity (U) & $0.3 \mathrm{~m} / \mathrm{s}$ \\
\hline Young's modulus of steel (E) & $210 \mathrm{Gpa}$ & Air density (AD) - $\rho_{f}$ & $1.2 \mathrm{~kg} / \mathrm{m}^{3}$ \\
\hline Density of steel - $\rho_{s}$ & $7800 \mathrm{~kg} / \mathrm{m}^{3}$ & Wing aspect ratio & 3 \\
\hline Reynolds number (chord) & 30,000 & $\begin{array}{l}\text { Normalized plunge amplitude } \\
\text { (w.r.t. chord) }-\mathrm{h}_{\mathrm{r}}\end{array}$ & 0.175 \\
\hline
\end{tabular}

Results for the flexible wing based at air density $\mathrm{AD}, 10 \mathrm{xAD}$, and 416xAD are presented later in the paper.

\section{Rigid wing in pure plunge}

The Reynolds number (based on chord) is set to 30,000. The flow was expected to be turbulent at this condition. To study the effect of transition and turbulence to the thrust and flow structure, the $k-\varepsilon$ models with/without filter were utilized in the computation to contrast with the results corresponding to laminar flow assumption. The grid size used in the computation is $120 \times 56 \times 60$ and the time-step size is 5 e- 3 s. Numerical experiments indicated that these parameters are suitable to obtain enough accuracy. The turbulence intensity was set to $4 \%$ at the inlet in the two turbulence cases. The filter size in the filter model was set to $6 \mathrm{e}-3 \mathrm{~m}$ which corresponds to the average cell size.

Fig. 5 includes the thrust coefficient results of three different computational cases compared with the experiment results. It may be observed that computational results have a decent agreement with those of experiment. It may also be observed that the inclusion of turbulent models did not have a significant effect on the response. The experimental data shows asymmetric thrust in the two strokes of each cycle, while the computational results show no distinguished difference between the two strokes. Compared to the experimental data, the computational results agree with the experimental data very well at the small force peaks, if the asymmetric difference is neglected. 

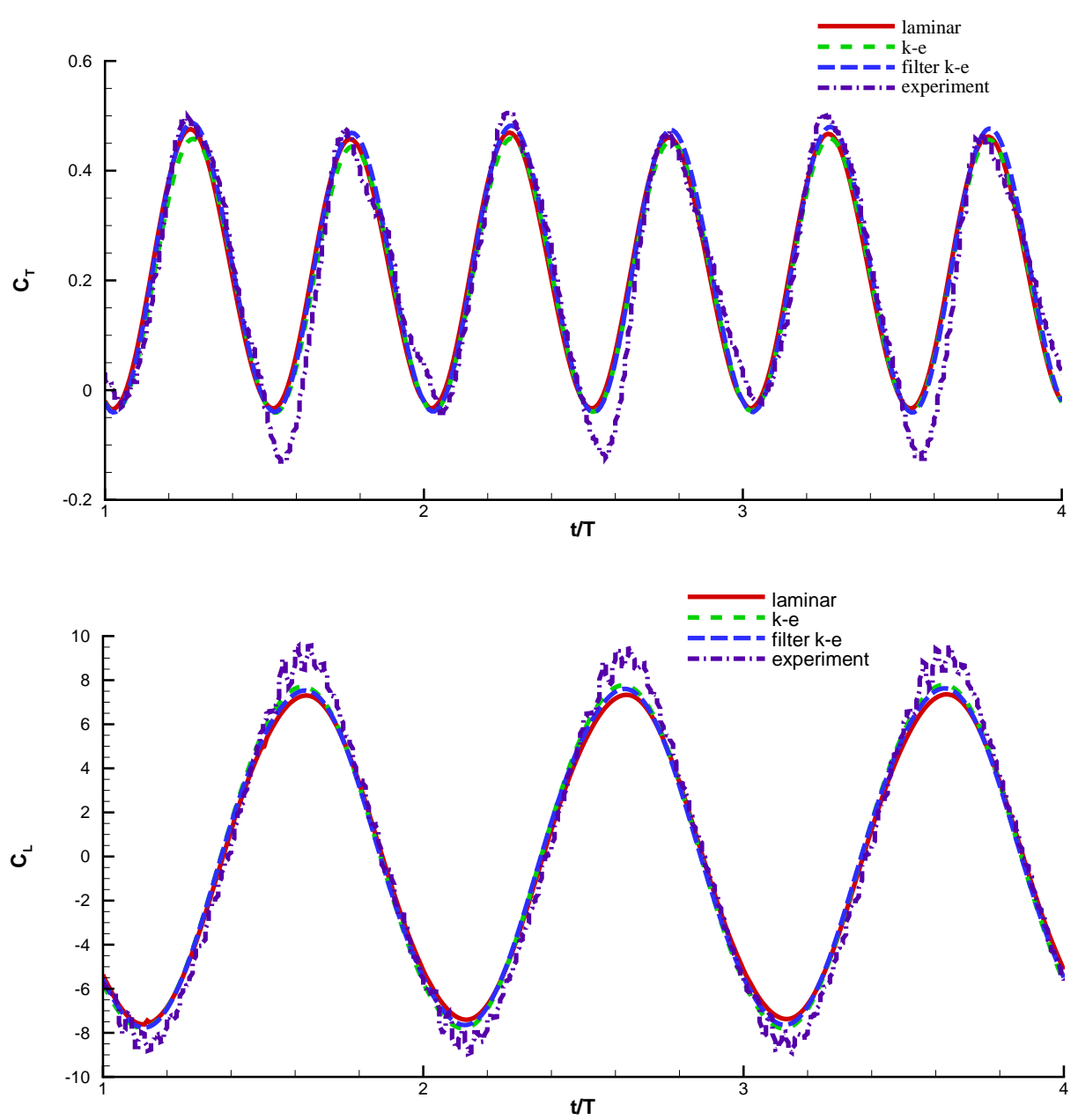

Fig 5: Thrust (upper) and Lift (lower) coefficients of experimental and computational results of rigid wing. $k-\varepsilon$ model, $k-\varepsilon$ with filter model and laminar flow are employed in the computation. $\left(\operatorname{Re}=30,000, h_{r}=0.175, k=1.82\right)$

To probe this further, the pressure distribution and stream lines at the mid-span station at four time instants in a cycle for the three different computations are plotted in Fig. 6 (in the body frame). The pressure distribution has the same pattern in all the three cases which explains the force histories being so close to each other (the viscous forces are of negligible contribution here). It was noticed that in the laminar and filter-based $k-\varepsilon$ cases, the separation bubbles existed on the wing surface; the size and the position of separation bubble were slightly different between the two computations. The computation using the original $k-\varepsilon$ model did not show the separation bubble in each of the four time instants because of the larger eddy viscosity. Nevertheless, the impact of the separation bubbles on aerodynamic forces is small. To further illustrate the entire flow field, Fig. 7 plots the streamlines along with pressure distribution on the wing surface at $\mathrm{t}=0.5 \mathrm{~T}$. The laminar and filter-based models clearly indicate the separation vortices on the upper surface, while in the $k-\varepsilon$ model computational result, no separation was observed on the whole wing. 
A.

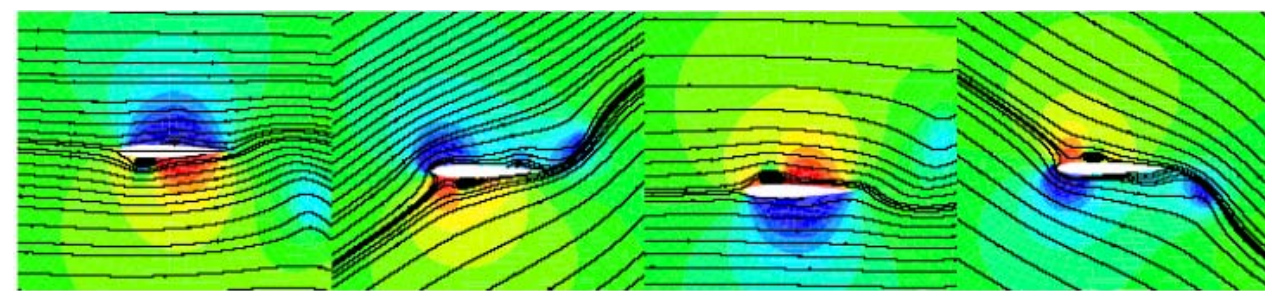

B.

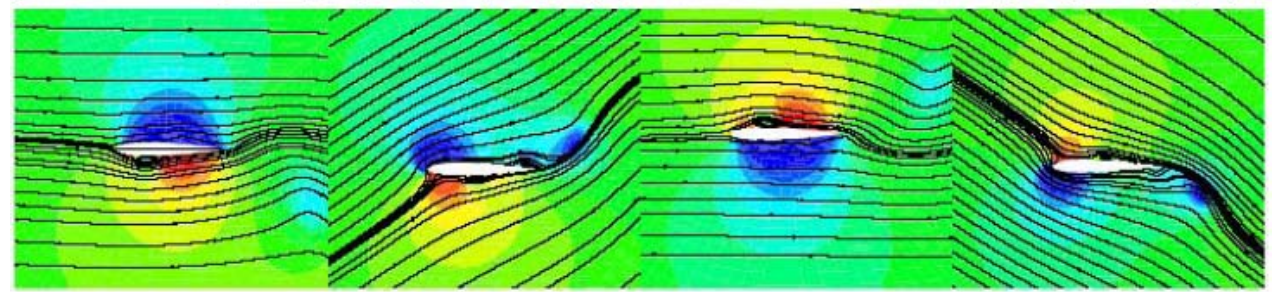

C

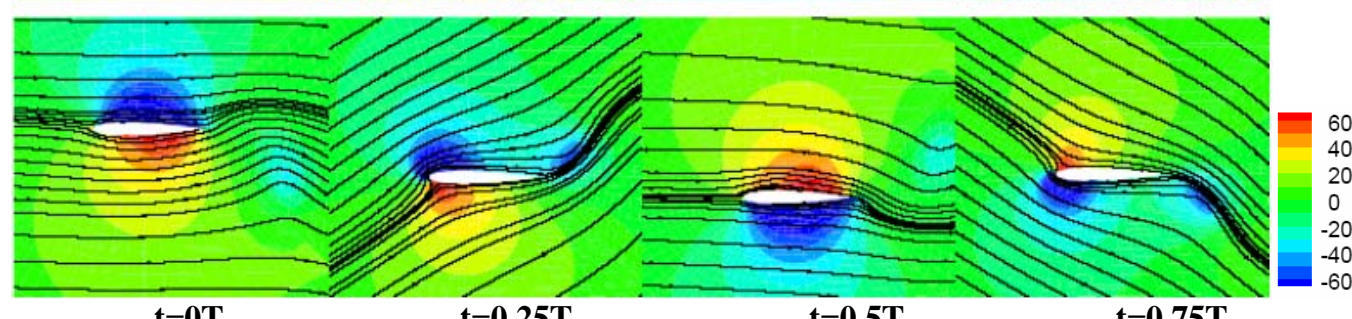

$\mathbf{t}=\mathbf{0} \mathbf{T}$

$\mathbf{t}=\mathbf{0 . 2 5 T}$

$\mathbf{t}=\mathbf{0 . 5 T}$

$\mathbf{t}=\mathbf{0 . 7 5 T}$

Fig 6: Flow structure and pressure distribution at mid wing-span station at

four time instants in one cycle

( A. laminar flow, B. filter model with filter size 6e-3 m, C. $k-\varepsilon$ model)

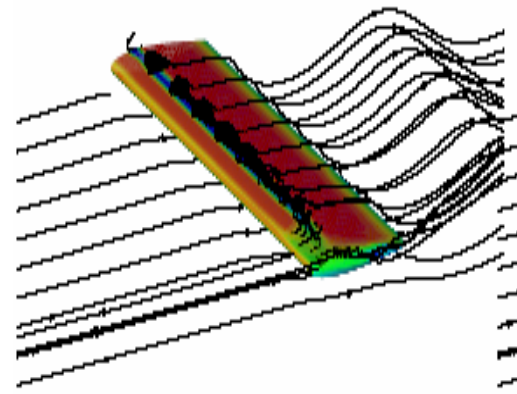

A

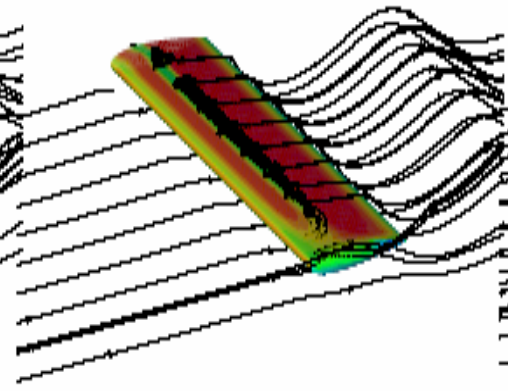

B

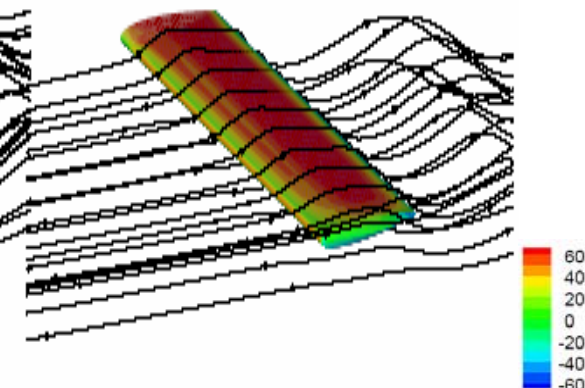

C

Fig 7: Flow structure and pressure distribution on the wing surface at $t=0.5 T$

( A. laminar flow, B. filter model with filter size 6e-3 m, C. $k-\varepsilon$ model)

\section{Flexible wing in pure plunge}

For flexible wing computations, the Navier-Stokes equations with no additional turbulence closure models are solved. Wing flexibility was included to qualitatively assess important characteristics like the induced phase lag between the responses of the rigid and the flexible wing and the contribution of elastic span-wise bending displacements to the overall thrust coefficient response of the wing. Flapping wings, in general, produce both aerodynamic/added mass and inertial/elastic forces. While these forces cause the wing to deform, the aerodynamic forces provide damping to the structure (in the absence of any structural damping 
which is the case here). In order to isolate the effects of aerodynamics on the structural deformation, flexible wing computations were done at three different flow densities while all other conditions were kept the same.

Fig. 8 includes the elastic tip deformation response (in the body frame) of the flexible wing in pure plunge at 3 different flow densities (air density, 10xair density, and 416xair density) compared to the case with no aerodynamic forcing. The body frame is defined as the one with prescribed plunge motion. It may be observed that as the flow density increases, the high frequency components of bending are damped out. This could be attributed to the increased aerodynamic damping at higher flow densities.

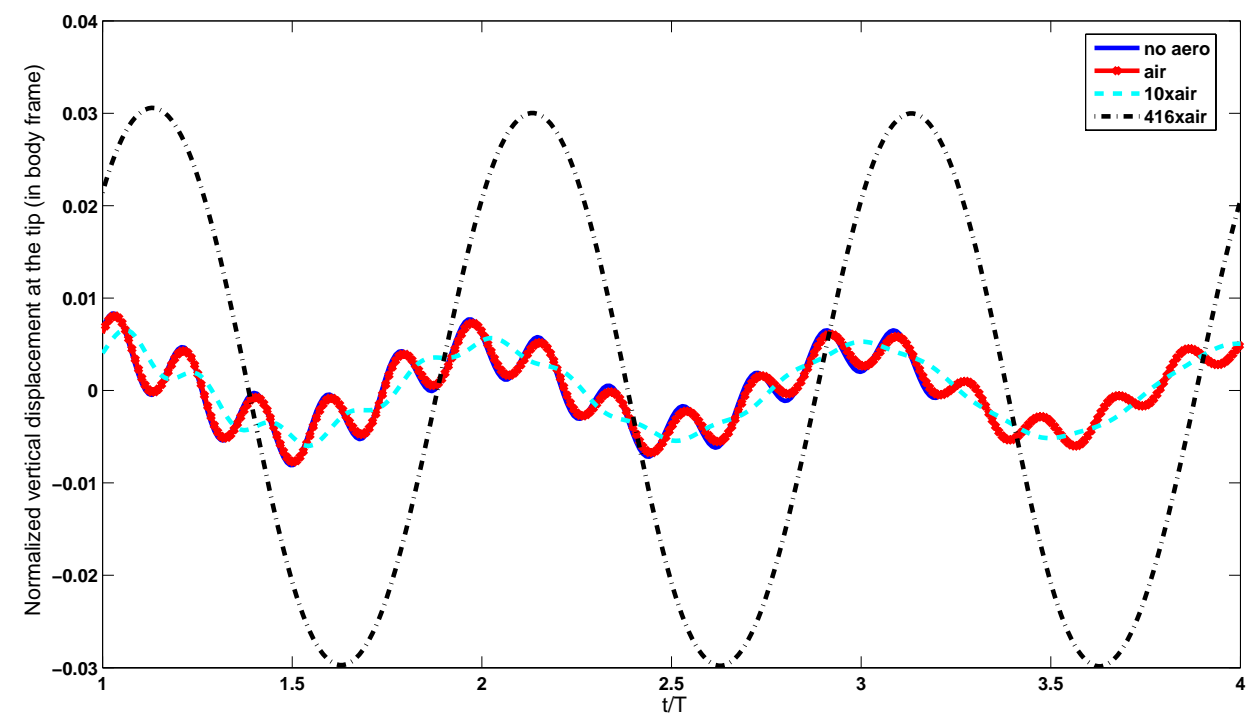

Fig 8: Tip displacements of the flexible wing at three different flow densities (body frame)

The tip displacement response of this wing with respect to the inertial frame is included in fig. 9 and compared with experimental response of a heavier fluid. As seen from the plot, the phase lag of the tip displacements relative to the flapping motion kept increasing with flow density. Although qualitative, it may also be inferred from the computational responses at lower flow densities that they all capture the trend in the experimental response in both amplitude and phase. This was further corroborated by doing a linear extrapolation of the displacement responses at lower densities to estimate the computational response at 833xair density which appears as 833xair density (extrapolated) in fig. 9. The extrapolated computational and experimental responses are seen to have a decent agreement in both phase and amplitude.

Fig. 10 includes the thrust coefficient response of the flexible wing at 3 different flow densities (air density, 10xair density, and 416xair density) compared to the computational and experimentally determined responses of the rigid wing at the same Reynolds number. Once again, the effect of induced phase lag is seen here. Incremental changes to the thrust coefficient are clearly seen with increasing flow density. Also, 
it may be noted that spanwise flexibility has proven to be beneficial to the thrust coefficient just as seen in the experiment.

Fig. 10 also includes the experimental response of the flexible wing for the heavier fluid case. Similar inferences as in the case of the displacement response may be drawn even in this case.

As sample results, the pressure and stream lines at mid-span station at four different time instants are shown in Fig 11 and Fig 12 respectively for the $413 \times$ AD case. It may be noted that there is a non-zero flow velocity on the wing surface in this case by virtue of elastic deformation and hence the streamline contours are different than those observed in the rigid wing case (Figs 6 and 7).

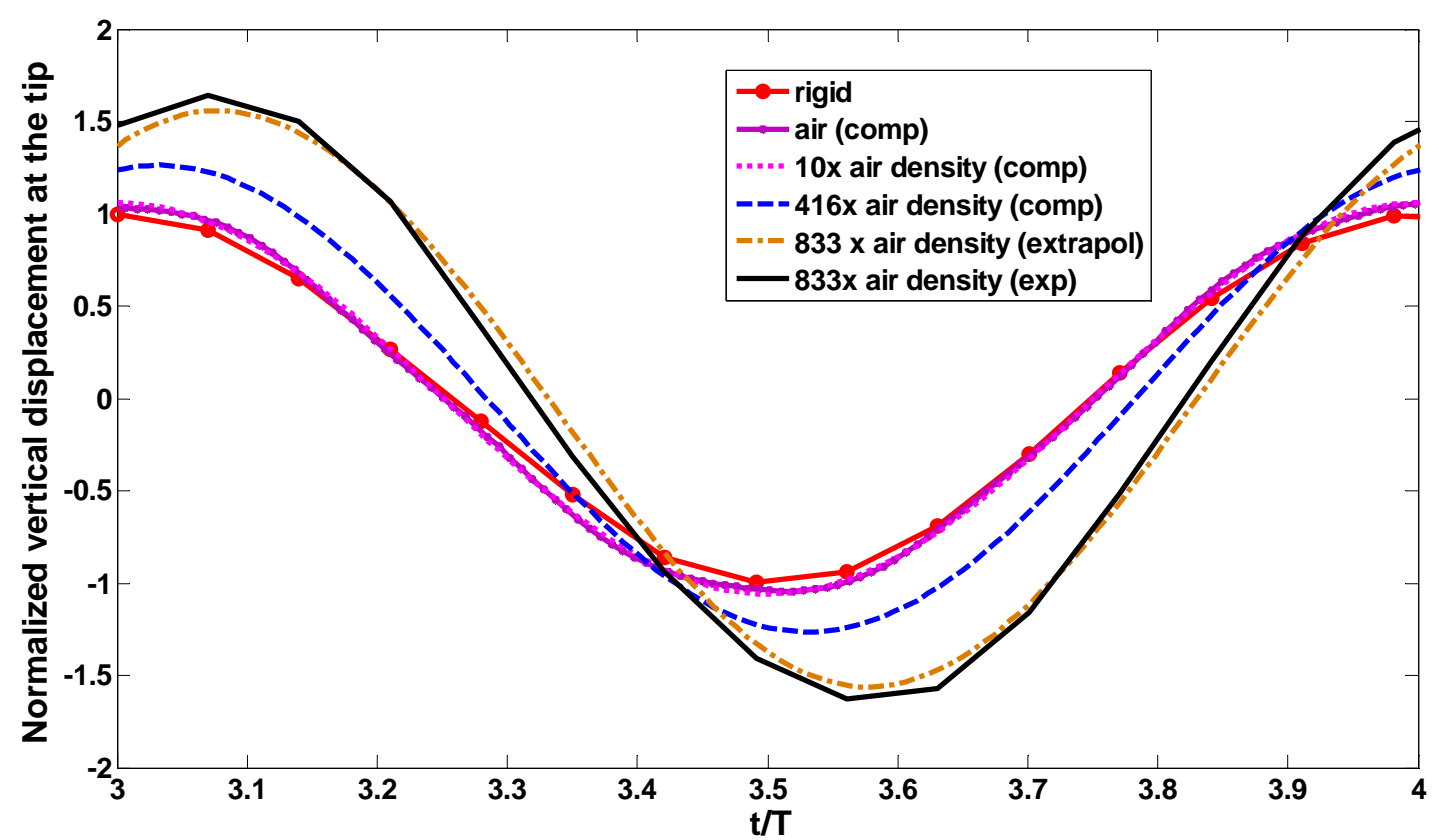

Fig 9: Tip displacement response of the flexible wing at three different flow densities (inertial frame) 


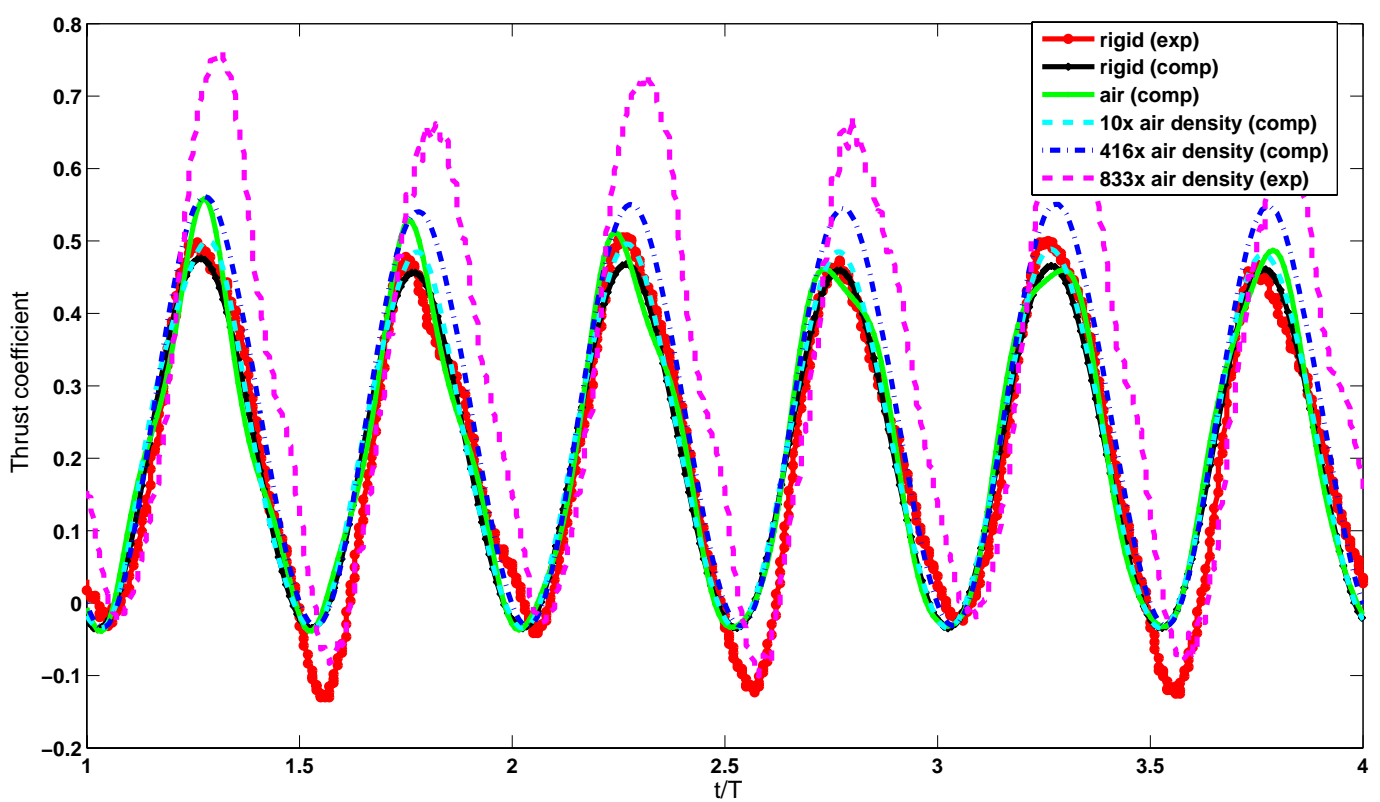

Fig 10: Thrust coefficient response of the flexible wing at three different flow densities

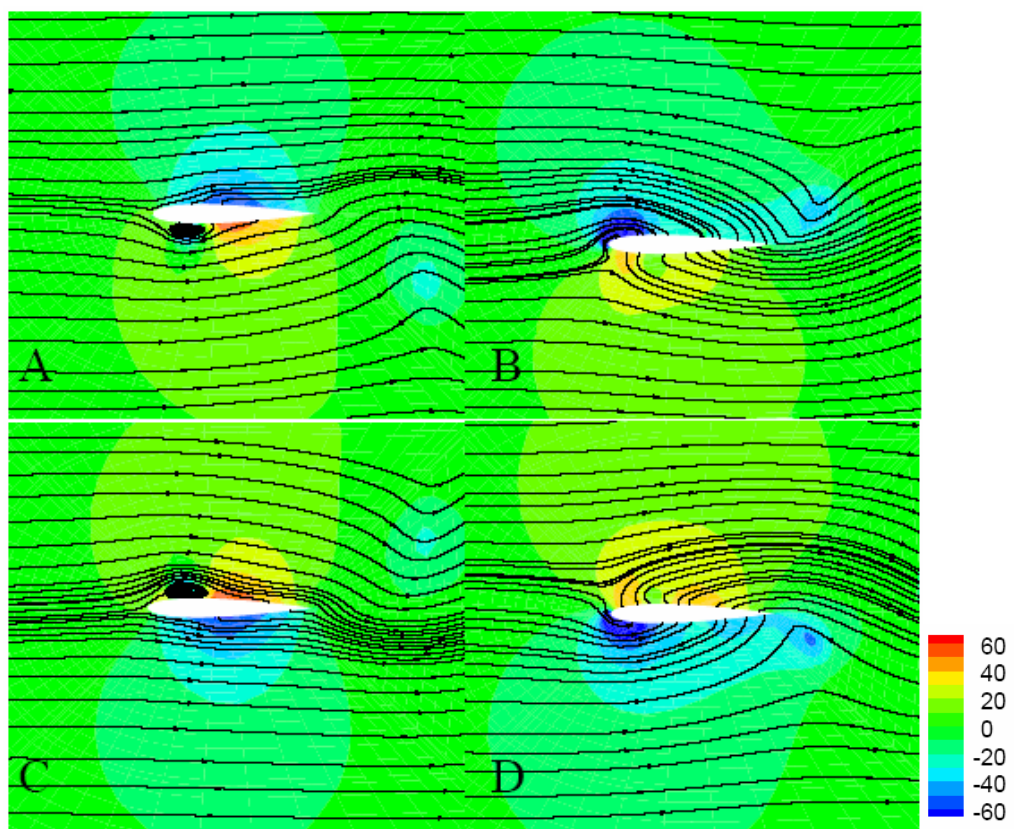

Fig 11: 2D flow structure and pressure distribution at mid wing-span station at four time instants $(A: t=0.0 T, B: t=0.25 T, C: t=0.5 T, D: t=0.75 T)$ in one cycle $(413 \times A D$ case) 

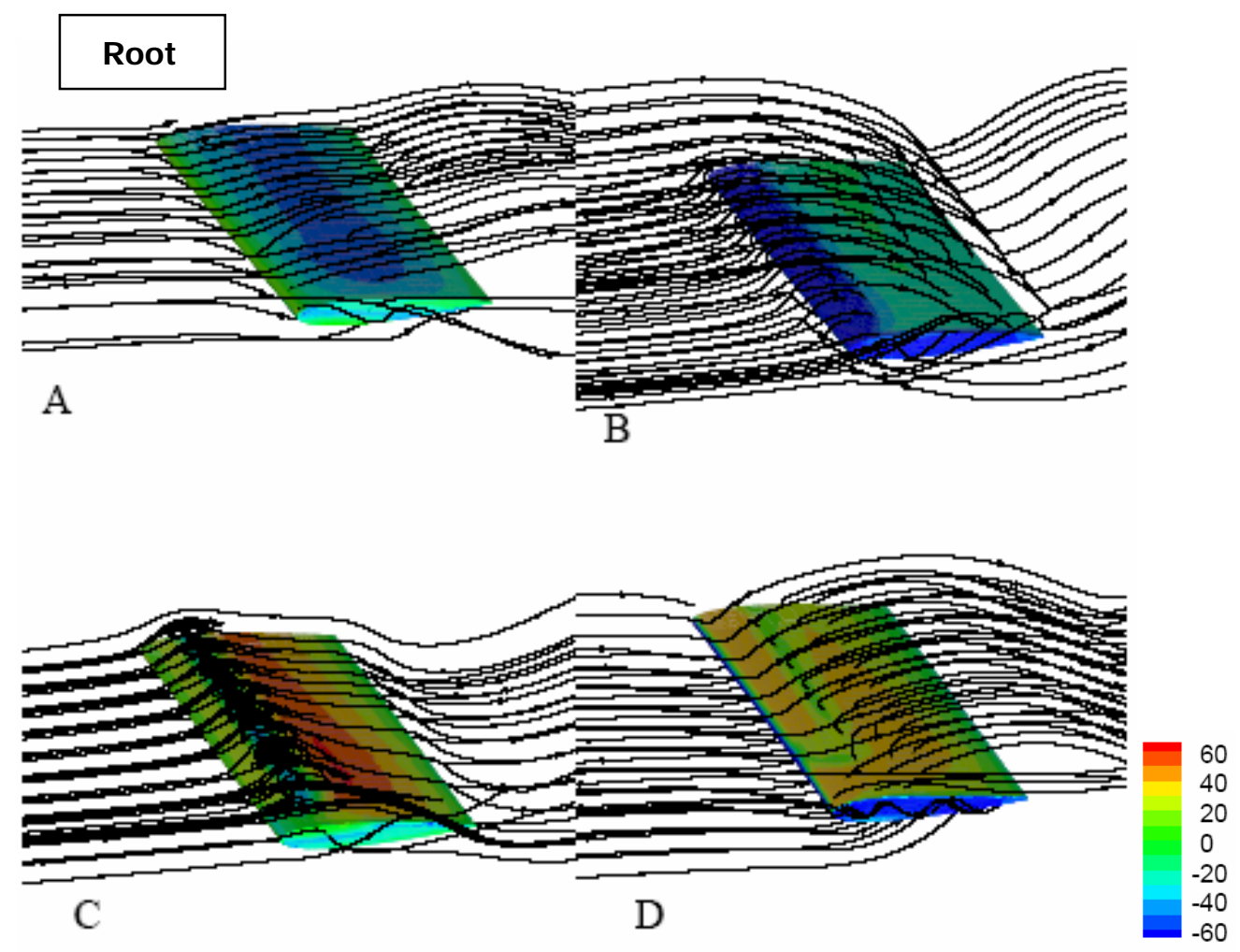

Fig 12: 3D flow structure and pressure distribution on the wing surface at time instants (A: $t=0.0 T, B: t=0.25 T, C: t=0.5 T, D: t=0.75 T)$ (413XAD case)

\section{Summary and Conclusion}

A computational fluid-structure framework for flapping wing MAV problems is presented. The fluid model includes laminar, the $\mathrm{k}-\varepsilon$ turbulence closure, and a filter-based $\mathrm{k}-\varepsilon$ closure. The structural model is based on an asymptotic approximation to the equations of elasticity. Using the slenderness as the small parameter, the equations are decomposed into two independent variational problems, corresponding to (i) crosssectional, small-deformation and (ii) longitudinal, large deformation analyses. The CFD and CSD solvers are coupled via an interface treatment enabling transmission of the aerodynamic loading from CFD to CSD, and of the shape deformation from CSD to CFD.

Based on Heathcote et al.'s experiment ${ }^{18}$, numerical simulations were conducted on a rectangular NACA0012 wing oscillating in pure heave. Quantitatively reasonable agreement with the experimental results was obtained for the thrust coefficient results on the rigid wing while the results on the flexible wing agree only qualitatively. Phase lag in the tip displacement was observed between the rigid and the flexible wing response in tune with the experiment. The amplitudes of elastic tip displacements and the induced phase lag increased with increasing flow density. 


\section{Acknowledgments}

The authors acknowledge the assistance of Dr. Dragos Viieru and Mr. Chang-Kwon-Kang of the University of Michigan, at various stages in this work. Thanks are also due to Prof. Gursul and Mr. Sam Heathcote of the University of Bath, United Kingdom for providing experimental data. This work was supported by the US Air Force under a MURI project and a collaborative center agreement. 


\section{References}

${ }^{1}$ Weis-Fogh, T., "Quick Estimate of Flight Fitness in Hovering Animals, Including Novel Mechanisms for Lift Production," Journal of Experimental Biology, Vol. Vol. 59, 1973, pp. 169-230.

${ }^{2}$ Ellington, C.P., Berg, C.v.d., Willmott, A.P., and Thomas, A.L.R., "Leading-Edge Vortices in Insect Flight," Nature, Vol. Vol. 384, 1996, pp. 626-630

${ }^{3}$ Dickinson, M.H., Lehmann, F.-O., and P.Sane, S., "Wing Rotation and the Aerodynamic Basis of Insect Flight," Science, Vol. 284, 1999, pp. 1954-1960.

${ }^{4}$ Van den Berg, C. and Ellington, C.P., "The Three-Dimensional Leading-Edge Vortex of a 'Hovering' Model Hawkmoth," Phil. Trans. R. Soc. Lond. B, Vol. 352, 1997, pp. 329-340.

${ }^{5}$ Liu, H. and Kawachi, K., "A Numerical Study of Insect Flight," Journal of Computational Physics, Vol. Vol. 146, 1998, pp. 124-156.

${ }^{6}$ Shyy, W. and Liu, H., "Flapping Wings and Aerodynamic Lift: the Role of Leading-edge Vortices", AIAA Journal, Vol. 45(2), 2007, pp. 2819-2821.

${ }^{7}$ Sun, M. and Tang, J., "Unsteady Aerodynamic Force Generation by a Model Fruit Fly Wing in Flapping Motion," Journal of Experimental Biology, Vol. 205, 2002, pp. 55-70

${ }^{8}$ Ramamurti, R. and Sandberg, W., "A Three-Dimensional Computational Study of the Aerodynamic Mechanisms of Insect Flight," Journal of Experimental Biology, Vol. Vol. 205, 2002, pp. 1507-1518.

${ }^{9}$ Viieru, D., Tang, J., Lian, Y., Liu, H., and Shyy, W., "Flapping and Flexible Wing Aerodynamics of Low Reynolds Number Flight Vehicles," AIAA paper 2006-0503, 2006.

${ }^{10}$ Tang, J., Viieru, D., and Shyy, W., "Effects of Reynolds Number and Stroke Amplitude on Hovering Wing Aerodynamics," 45th Aerospace Sciences Meeting \& Exhibit, AIAA paper 2007-129, 2007.

${ }^{11}$ Wang, Z.J., Birch, J.M., and Dickinson, M.H., "Unsteady Forces and Flows in Low Reynolds Number Hovering Flight:Two-Dimensional Computations Vs Robotic Wing Experiments," Journal of Experimental Biology, Vol. 207, 2004, pp. 449-460.

${ }^{12}$ Freymuth, P., "Thrust Generation by an Airfoil in Hover Modes," Expts Fluid, Vol. 9, 1990, pp. 17-24.

${ }^{13}$ Shyy, W., Lian, Y., Tang, J., Viieru, D., Liu, H. Aerodynamics of Low Reynolds Number Flyers, Cambridge University Press, New York.

${ }^{14}$ S. A. Combes and T. L. Danial, "Flexural stiffness in insect wings I. Scaling and the influence of wing venation", J. Experimental Biology, Vol. 206, pp. 2927-2987.

${ }^{15}$ Wootton, R.J., Herbert, R.C., Young, P.G., and Evans, K.E., "Approaches to the structural modeling of insect wings," Philosophical transactions of the Royal society of London, Vol. 358, pp. 1577-1587.

${ }^{16}$ Heathcote, S., Martin, D., and Gursul, I., "Flexible Flapping Airfoil Propulsion at Zero Freestream Velocity," AIAA Journal, Vol. 42, No. 11, 2004, pp. 2196-2204.

${ }^{17}$ Heathcote, S., and Gursul, I., "Flexible Flapping Airfoil Propulsion at Low Reynolds Numbers," AIAA Paper 2005-1405, Jan. 2005.

${ }^{18}$ Heathcote, S., Z, Wang., and Gursul, I, "Effect of Spanwise Flexibility on Flapping Wing Propulsion”, AIAA Paper 2006-2870, Jan. 2005..

${ }^{19}$ Tang, J., Viieru, D., and Shyy, W., “A Study of Aerodynamics of Low Reynolds Number Flexible Airfoils," $37^{\text {th }}$ AIAA Fluid Dynamics Conference and Exhibit, Miami, FL, 25-28 June 2007, AIAA Paper No. 2007-4212.

${ }^{20}$ Launder, B.E., Spalding, D.B, “The numerical computation of turbulent flows", Comp. Meth. Appl. Mech. Eng. Vol. 3, 1974, pp. 269-289.

${ }^{21}$ Johansen, S.., Wu, J., Wei, Shyy, "Filter-based unsteady RANS computations", International Journal of Heat and fluid flow, Vol. 25, 2004, pp. 10-21.

${ }^{22}$ Patankar, S.V. and Spalding, D.B., "A Calculation Procedure for Heat, Mass and Momentum Transfer in Three-Dimensional Parabolic Flows," Int. J. Heat Mass Transfer, Vol. 15, 1972, pp. 1787-1806.

${ }^{23}$ Shyy, W., "Computational Modeling for Fluid Flow and Interfacial Transport," edited, Amsterdam, The Netherlands, Elsevier. 1994.

${ }^{24}$ Lian, Y., and Shyy, W., "Numerical Simulations of Membrane Wing Aerodynamics for Micro Air Vehicle Applications," Journal of Aircraft, Vol. 42, No. 4, 2005, pp. 865-873. 
${ }^{25}$ Thomas, P.D. and Lombard, K., "Geometric Conservation Law and Its Application to Flow Computations on Moving Grids," AIAA Journal, Vol. 17, 1979, pp. 1030-1037.

${ }^{26}$ Kamakoti, R. and Shyy, W., "Fluid-Structure Interaction for Aeroelastic Applications," Progress in Aerospace Sciences, Vol. 40, 2004, pp. 535-558.

${ }^{27}$ Palacios, R., Cesnik, C.E.S., "Cross-Sectional Analysis of Non-Homogenous Anisotropic Active Slender Structures," AIAA Journal vol. 43(12), 2005, pp. 2624-2638.

${ }^{28}$ Palacios, R., Cesnik C.E.S., "A Geometrically-Nonlinear Theory of Active Composite Beams with

Deformable Cross Sections" (Accepted for publication in the AIAA Journal, 2008)

${ }^{29}$ Smith, Marilyn J., Hodges, Dewey., Cesnik, C.E.S., "Evaluation of Computational Algorithms Suitable

For Fluid-Structure Interactions," Journal of Aircraft, Vol. 37, No. 2, 2000, pp. 282-294. 